\title{
Space-Time Autocoding Constellations with Pairwise-Independent Signals
}

\author{
Thomas L. Marzetta \\ Lucent Technologies \\ Babak Hassibi ${ }^{1}$ \\ Dept. of Electrical Engineering \\ Bertrand Hochwald \\ Lucent Technologies \\ 600 Mountain Avenue, Rm. 2C-373 California Institute of Technology 600 Mountain Avenue, Rm. 2C-363 \\ Murray Hill, NJ 07974 \\ Pasadena, CA 91125 \\ e-mail: \\ Murray Hill, NJ 07974 \\ e-mail: \\ tlm@research.bell-labs.com \\ hassibi@systems.caltech.edu hochwald@research.bell-labs.com
}

Abstract - The space-time autocoding effect implies that arbitrarily reliable communication is possible within a single coherence interval in Rayleigh flat fading as the symbol-duration of the coherence interval and the number of transmit antennas grow simultaneously. For relatively short (e.g., 16-symbol) coherence intervals, a codebook of isotropically random unitary space-time signals theoretically supports transmission rates that are a significant fraction of autocapacity with an extremely low probability of error. However a constellation of the required size (typically $L=2^{80}$ ) is impossible to generate and store, and due to lack of structure there is little hope of finding a fast decoding scheme. We propose a random, but highly structured, constellation that is completely specified by $\log _{2} L$ independent isotropically distributed unitary matrices. The distinguishing property of this construction is that any two signals in the constellation are pairwise statistically independent and isotropically distributed. Thus, the pairwise probability of error, and hence the union bound on the block probability of error, of the structured constellation is identical to that of a fully random constellation of independent signals.

A full version of this paper may be downloaded from the web site http://mars.bell-labs.com

In the course of this work, we provide a novel proof of the little-known fact that any integer power (greater than one) of an isotropically random unitary matrix (also called Haar measure) is not isotropically random, with one exception: a one-by-one complex unitary matrix. In Figure 1, this effect can be seen in powers of a $3 \times 3$ real isotropically random orthogonal matrix.

\footnotetext{
${ }^{\mathbf{1}}$ Professor Hassibi left Lucent Technologies and joined Caltech in January.
}
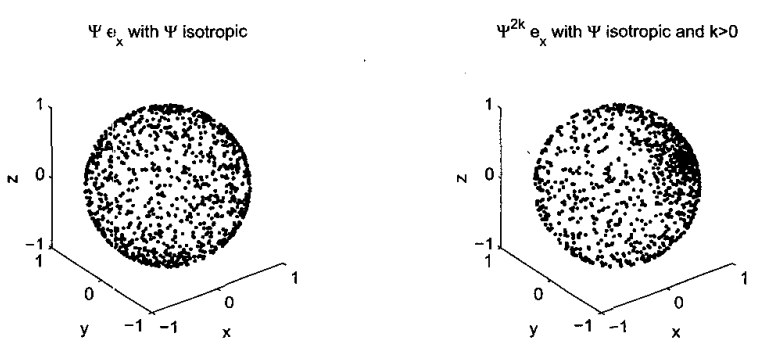

$\Psi^{2 k+1} e_{x}$ with $\Psi$ isotropic and $k>0$

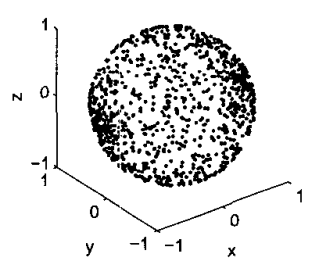

Figure 1: Powers of a $3 \times 3$ real, isotropically distributed orthogonal matrix $\Psi$ are not isotropically distributed. Upper left: single application of random rotation to the unit vector $e_{x}$ results in a unit vector that is equally likely to lie anywhere on the unit sphere. Upper right: an even number of applications of the same random matrix is biased towards $e_{x}$. Lower left: an odd number of applications of the same random matrix is biased towards $\pm e_{x}$. 